\title{
Oil Mill Wastewater and its Effects
}

\author{
Serafina Andiloro* \\ Department of AGRARIA, Mediterranea University of Reggio Calabria, Italy
}

*Corresponding author: Serafina Andiloro, Mediterranea University of Reggio Calabria, Department of AGRARIA, Località Feo di Vito, I-89122 Reggio Calabria, Italy.

Received Date: July 15, 2019

Published Date: July 25, 2019

\section{Short Communication}

Olive mill wastewater (OMW) is the liquid by-product generated during olive oil production. Different papers have discussed the effects of OMW discharge on soil characteristics, plant production and phytotoxicity.

OMW are characterized by high organic load, concentrations of total suspended solids and phenolic compounds, and depends on the olives degree of ripening and the post-harvest, storage conditions, oil and grease. The relationship between soil and OMW is also in dependence of their quantity and qualitative chemical composition. It is noteworthing that the quantity and the quality of OMW is influenced by the olive oil extraction system. In a so called three phases system 50-70 L of lukewarm water are added per 100 $\mathrm{kg}$ olive fruits in the horizzontal centrifuge (decanter), whereas 0-20 L of lukewarm water are added per $100 \mathrm{~kg}$ olive fruits in a so called tw o phases system. As a consequence a three phases system produces larger OMW volumes (93.6-95.0 L per $100 \mathrm{~kg}$ olive fruits) than a two phases system (6.1 L per $100 \mathrm{~kg}$ olive fruits). The phenolic content in OMW is related to the water added during the oil extractive process, in fact the higher water adding in the three phases system dilutes phenols (197 mg/L) in larger OMW volumes whereas a higher phenol content (292 mg/L) is found in OMW from a two phases system. A 1.2-1.4 kg oil aliquot per $100 \mathrm{~kg}$ olives is also contained in the OMW. Olive oil is mainly composed by glycerides (about 98.5\%) and minor components (about 1.5\%) such as phenols, sterols, fatty alcohols, and waxes whose composition varies during olive ripening. For this reason the OMW composition after an olive oil extraction on October is different with respect OMW after an olive oil extraction on December from drupes even if produced in the same olive orchard and in the same harvest year.

For these reasons, increasing attention has been given to find the best methods to spread OMW on agricultural lands and to recycle both the organic matter and the nutritive elements in the soil crops system. Moreover, agricultural irrigation with wastewater effluents became a common practice in arid and semiarid regions, where it was used as a readily available and inexpensive option to fresh water. The effects of OMWs on crops and soils are reviewed. Generally, positive effects of the soil have been observed with an increase in the porosity and stability of the aggregates. Since these effects are due to the content of organic substance. A temporary reduction in the soil infiltration rate was detected immediately after OMW irrigation, however followed by a significant temporal increase after three weeks. In general, due to the variability of OMW application rates and properties of receiving soils, literature data on OMW effects on soil properties is not unanimous and in many cases even contradictory. The components and residues of oil and grease are wax-like substances that form a coating on soil particles.

Soil hydrophobicity, also known as soil water repellency (hereinafter "WR"), represents the situation whereby the soil does not wet when water is spontaneously applied. This phenomenon induces several soil issues, such as a reduction of the infiltration capacity (retarding, specifically, surface water infiltration, Brandt, 1969), the increase of the overland flow and soil erosion, the development of fingered flow in structural or textural preferential flow pathways, and the creation of unstable, irregular wetting fronts.

In addition, most of the studies reported in the scientific literature has mainly evaluated the long-term that is, at yearly or decade scales - effects of OMW land spreading on soil properties with contrasting results.

In many field crops, the application of OMWs has beneficial effects on crop yield. However, the application of OMWs must adequately precede sowing to be beneficial. Based on the literature review, it appears that seed germination is the most sensitive phase for OMW application. The response of different species during germination appears to result from the high OMW polyphenol content. The polyphenol content was young plantlets in the 
examined species. In addition, osmotic stress, which was induced by the high salinity of the OMWs, was toxic for young plantlets. This effect is also species dependent.

\section{Acknowledgement}

None.

\section{Conflict of Interest}

No conflict of interest. 